\title{
Fas palmitoylation by the palmitoyl acyltransferase DHHC7 regulates Fas stability
}

\author{
A Rossin ${ }^{1}$, J Durivault ${ }^{1}$, T Chakhtoura-Feghali ${ }^{1}$, N Lounnas ${ }^{1}$, L Gagnoux-Palacios ${ }^{1}$ and A-O Hueber ${ }^{*}, 1$
}

The death receptor Fas undergoes a variety of post-translational modifications including S-palmitoylation. This protein acylation has been reported essential for an optimal cell death signaling by allowing both a proper Fas localization in cholesterol and sphingolipid-enriched membrane nanodomains, as well as Fas high-molecular weight complexes. In human, S-palmitoylation is controlled by 23 members of the DHHC family through their palmitoyl acyltransferase activity. In order to better understand the role of this post-translational modification in the regulation of the Fas-mediated apoptosis pathway, we performed a screen that allowed the identification of DHHC7 as a Fas-palmitoylating enzyme. Indeed, modifying DHHC7 expression by specific silencing or overexpression, respectively, reduces or enhances Fas palmitoylation and DHHC7 co-immunoprecipitates with Fas. At a functional level, DHHC7-mediated palmitoylation of Fas allows a proper Fas expression level by preventing its degradation through the lysosomes. Indeed, the decrease of Fas expression obtained upon loss of Fas palmitoylation can be restored by inhibiting the lysosomal degradation pathway. We describe the modification of Fas by palmitoylation as a novel mechanism for the regulation of Fas expression through its ability to circumvent its degradation by lysosomal proteolysis.

Cell Death and Differentiation (2015) 22, 643-653; doi:10.1038/cdd.2014.153; published online 10 October 2014

Fas (CD95, TNFRSF6) is a prototypic death receptor that belongs to the tumor necrosis factor receptors (TNFRs) superfamily. ${ }^{1}$ Upon activation by the Fas ligand (FasL, TNFSF6), Fas initiates a signaling cascade that leads to caspases activation and ultimately to cell death. ${ }^{2}$ For these reasons, Fas was mainly considered as a tumor-suppressor protein. However, accumulating evidences support a significant role for Fas in alternative non-death signaling leading to cell survival, proliferation, epithelial-mesenchymal transition, cancer growth and metastasis in some context. ${ }^{3-5}$ Such conditional multi-signaling of Fas has been well demonstrated in colon cancer model. 6,7

We demonstrated that Fas and FasL are constitutively modified by S-palmitoylation, a reversible post-translational modification that consists in the addition of a palmitic acid on the cysteine residue through a thiol linkage. ${ }^{8-10}$ The palmitoylation of a protein can affect its interactions with its surrounding membrane lipids and proteins, therefore impacting certain properties such as association with specific membrane domains, trafficking between cell compartments or stability. ${ }^{11-13}$ The palmitoylation reaction is catalyzed by the conserved DHHC (aspartate-histidine-histidine-cysteine) family of enzymes, which are characterized by the specific DHHC motif required for the palmitoyl acyltransferase (PAT) activity. ${ }^{14}$ In human, the 23 members of the DHHC family described are localized to the distinct intracellular membrane compartments, mainly the Golgi apparatus and the endoplasmic reticulum where palmitoylation likely occurs. ${ }^{14,15}$ Since their discovery in 2002, increasing numbers of DHHC-substrate pairs have been identified allowing a deeper comprehension of palmitoylation regulation. ${ }^{16}$

Fas palmitoylation occurs on an intracellular cysteine adjacent to the transmembrane domain (cysteine 199 in human) and is critical for its ability to trigger cell death. ${ }^{8,9}$ At the molecular level, we and others already reported that the pro-death role of Fas palmitoylation relies on (i) the formation of supramolecular Fas aggregates ${ }^{9}$ and (ii) Fas targeting in nanodomains enriched in cholesterol and glycosphingolipids, which is a prerequisite for proper activation of Fas-induced cell death. ${ }^{8,17-21}$ In these domains and upon FasL binding, palmitoylation allows Fas to recruit ezrin and actin cytoskeleton that lead to Fas internalization and transmission of death signal. ${ }^{8}$ We thus aimed at understanding how Fas palmitoylation is regulated. We report here the identification of $\mathrm{DHHC7}$ as a Fas-palmitoylating enzyme and revealed an additional role of Fas palmitoylation. The latter consists in the stabilization of Fas by preventing its degradation through the lysosomal pathway.

\section{Results}

Fas palmitoylation is required for optimal cell death in colorectal cancer (CRC) cells. So far, endogenous Fas palmitoylation has only been demonstrated in the lymphoid system. $^{8,9,22}$ We submitted several colorectal cell lines to the acyl-biotin exchange (ABE) technic, which assess the protein palmitoylation status of proteins and show that Fas is palmitoylated in epithelial cells from CRC origin (Figure 1a).

'Université de Nice, Institut de Biologie Valrose, CNRS UMR 7277, INSERM UMR 1091, Nice, France

*Corresponding author: A-O Hueber, Université de Nice, Institut de Biologie Valrose, CNRS UMR 7277, INSERM UMR 1091, Parc Valrose, Bâtiment des Sciences Naturelles, Nice 06108, France. Tel: +33 492 076447; Fax: +33 492 076170; E-mail: hueber@ unice.fr

Abbreviations: CRC, colorectal cancer; DHHC, aspartate-histidine-histidine-cysteine; PAT, palmitoyl acyltransferase; ABE, acyl-biotin exchange

Received 09.4.14; revised 24.7.14; accepted 18.8.14; Edited by S Kaufmann; published online 10.10.14 
a

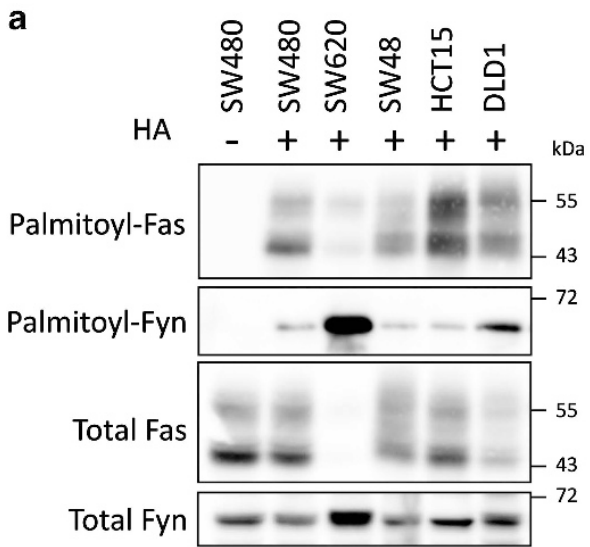

C

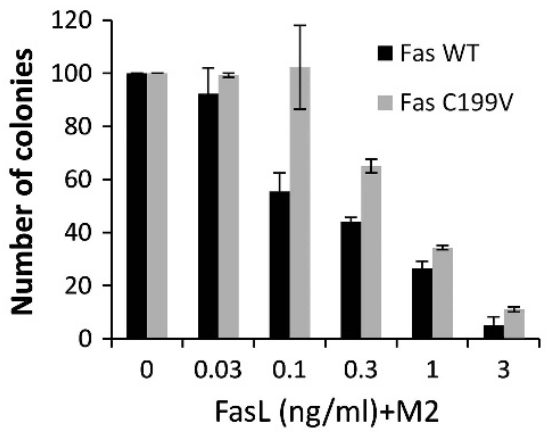

b

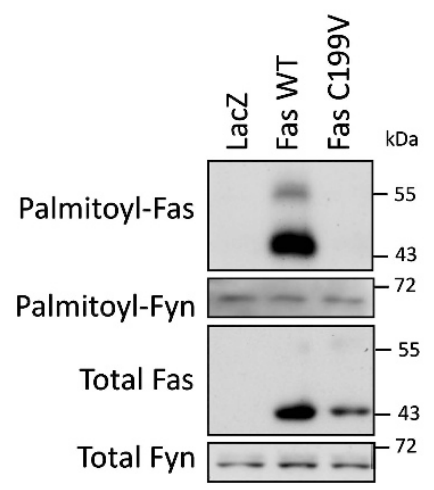

d

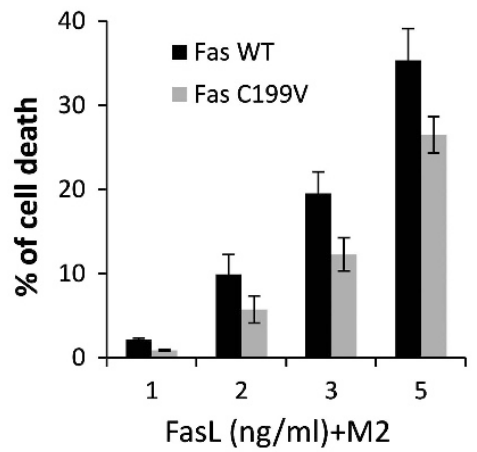

e

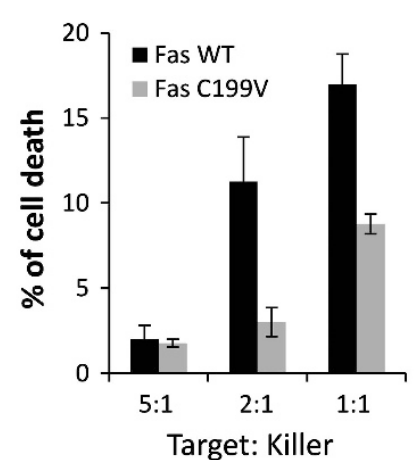

f

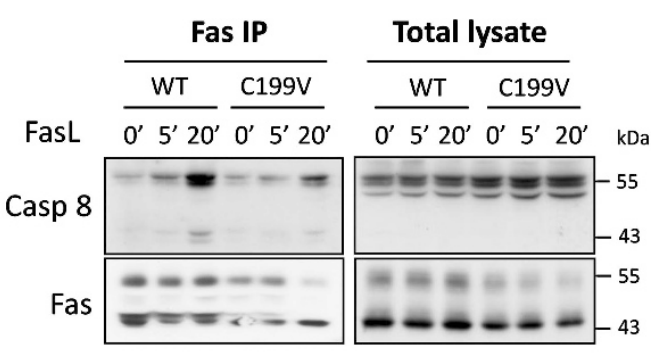

Figure 1 Fas palmitoylation is critical for optimal cell death in CRC cells. Fas palmitoylation status was determined by submitting the CRC cell lines SW480, SW620, SW48, HCT15 and DLD1 (a) or SW480 stably expressing LacZ, Fas WTor Fas C199V (b) to the acyl-biotin exchange (ABE) technic. The S-palmitoyl-thioester link is specifically cleaved by hydroxylamine (HA) treatment and substituted by a biotin moiety. Biotinylated proteins are immunoprecipitated, separated by SDS-PAGE and immunoblotted with anti-Fas (a), anti-V5 (b) to assess exogenous Fas level and anti-Fyn antibodies, Fyn being used as an internal palmitoylated control. Controls without HA treatment are included to assess the specificity of the reaction. (c) Colony forming assay was performed on SW480 Fas WT and SW480 Fas C199V. The number of colonies was estimated upon 10 days exposure to the indicated concentration of rhFasL crosslinked with M2. Data are shown as mean + S.D. (d) Dose response of Fas-induced cell death in SW480 Fas WT and SW480 Fas C199V after $2 \mathrm{~h}$ of rhFasL treatment. Basal cell death was subtracted as background. Data are shown as mean \pm S.D. (e) SW480 Fas WT and SW480 Fas C199V were co-cultured for $5 \mathrm{~h}$ with the killer Reh cells stably expressing FasL and assessed for cell death. Basal cell death obtained from co-culture with Reh cells not expressing FasL was subtracted. Data are shown as mean \pm S.D. (f) Fas was immunoprecipitated from lysates of SW480 Fas WT and SW480 Fas C199V exposed for the indicated time to $10 \mathrm{ng} / \mathrm{ml}$ rhFasL+M2. Detection of the co-immunoprecipitated caspase 8 was analyzed by immunoblotting after SDS-PAGE resolution and Fas was detected with anti-V5 antibody

A classical Fas expression pattern is detected by western blot with several bands (mainly 2) comprised between 45 and $55 \mathrm{kDa}$ reflecting receptor modification such as glycosylation, thus showing that all these forms of Fas are palmitoylated.

As we previously identified the cysteine 199 (C199) of Fas as the unique palmitoylation site, we generated stable SW480 cells overexpressing either V5-tagged Fas wild type (Fas WT), V5-tagged Fas mutated on C199 (Fas C199V) or LacZ-V5, the latter representing a control with endogenous Fas expression (Supplementary Figure S1). We confirmed in this cellular context that Fas is palmitoylated only on cysteine 199 (Figure 1b). We then tested the ability of Fas C199V to respond to FasL stimulation by using different functional assays. First, the capacity of FasL to inhibit the formation of SW480 colonies is reduced when a palmitoylation-deficient mutant is expressed (Figure 1c). Mutating the palmitoylation site decreases SW480 cell death upon both exposure to crosslinked soluble FasL (Figure 1d) 
and to membrane-anchored FasL (Figure 1e). At a molecular level, the recruitment and activation of caspase 8 within the DISC is impaired when a palmitoylation-deficient mutant is expressed (Figure 1f). Therefore, Fas palmitoylation occurs in CRC cells and is contributing to optimal Fas-induced death signaling, as it was the case in lymphoid cells.

DHHC7 is a Fas-palmitoylating enzyme. In order to identify the enzyme(s) responsible for Fas palmitoylation, we used a 2-step screen in the HEK293 cells in which the DHHC family members were either overexpressed or silenced before submitting to $\mathrm{ABE}$. Overexpression of Fas with one of the 23 GFP-tagged DHHC family members established that 5 of them, DHHC1, 3, 7, 11 and 14 were able to increase Fas palmitoylation (data not shown). The silencing of each of the five preselected DHHC by RNAi strategy (two siRNA per $\mathrm{DHHC}$ ) demonstrated that targeting endogenous $\mathrm{DHHC7}$ expression significantly decreases Fas palmitoylation (Figure 2a). Knocking down endogenous DHHCs 1 and 11 only weakly decreases Fas palmitoylation, whereas knocking down DHHCs 3 and 14 has no effect. Thus, our results show that $\mathrm{DHHC7}$ is a main Fas-palmitoylating enzyme in HEK293 cells.

This result was confirmed in colorectal SW480 cells in which we modulated $\mathrm{DHHC} 7$ expression: knocking down $\mathrm{DHHC} 7$ by siRNA decreases Fas palmitoylation (Figure 2b), whereas overexpression of AcGFP-tagged $\mathrm{DHHC7}$ significantly increases it (Figure 2c). Notably, the overexpression of a catalytically inactive $\mathrm{DHHC} 7$ in which the cysteine 160 of the $\mathrm{DHHC}$ domain is mutated in serine does not affect Fas palmitoylation, which reinforce the conclusion of a specific and direct DHHC7 effect on Fas (Figure 2c). Immunoblotting with anti-DHHC7 antibody shows that this enzyme is expressed in all colorectal cell lines tested (Figure 2d). As reported by several studies, we confirmed by immunofluorescence that $\mathrm{DHHC7}$ is expressed in the Golgi apparatus ${ }^{15,23}$ (Supplementary Figure S2a), thus suggesting that Fas palmitoylation occurs in this vesicular compartment.

The palmitoylation reaction consists of a two-step mechanism in which a direct interaction between the $\mathrm{DHHC}$ and its substrate has been often reported. ${ }^{16,23-25}$ We show that endogenous $\mathrm{DHHC7}$ constitutively co-immunoprecipitates with endogenous Fas in SW480 LacZ cell lysate (Figure 2e). As expected, this $\mathrm{DHHC}$ /Fas association is enhanced when Fas WT is overexpressed (Figure 2e). In order to further characterize this interaction, DHHC7-AcGFP and DHHC7 C160S-AcGFP were transiently transfected in SW480 cells and a strong interaction is detected between endogenous Fas and DHHC7-AcGFP (Figure 2f). However, this association is much weaker with DHHC7 C160S-AcGFP, suggesting that a functional $\mathrm{DHHC}$ catalytic site is required for an optimal association (Figure 2f). In addition, an association is still detected with Fas $\mathrm{C} 199 \mathrm{~V}$ demonstrating that $\mathrm{DHHC} 7$ can also interact with non-palmitoylable Fas molecules (Supplementary Figure S2b). Altogether, our results show that $\mathrm{DHHC7}$ is a Fas-palmitoylating enzyme.

DHHC7-mediated palmitoylation regulates Fas expression and organization in membranes. We observed that knocking down the Fas-palmitoylating enzyme $\mathrm{DHHC7}$ significantly decreases Fas endogenous cell surface and total expression as demonstrated by flow cytometry, protein cell surface biotinylation and western blot analysis (Figure $3 a$ and Supplementary Figures S3b and c). In contrast, it does not affect the expression of the TNFR1, which is not modified by palmitoylation (Supplementary Figure S3a). Moreover, decreasing the expression of $\mathrm{DHHC}$, which is the closest palmitoyl transferase to $\mathrm{DHHC7}$ in terms of sequence and function, does not affect Fas expression (Figure 3a).

Importantly, knocking down DHHC7 expression in SW480 Fas WT cells also affects overexpressed Fas surface and total expression, whereas this effect is almost abrogated when Fas is mutated on its palmitoylation site (Figure $3 \mathrm{~b}$ ). This suggests that the decrease in Fas expression observed upon DHHC7 silencing depends mainly on the palmitoylation of Fas itself. Interestingly, Fas C199V level of expression is around $30 \%$ lower than Fas WT, reinforcing the idea that Fas palmitoylation is having a role in Fas expression (Figure $3 b$ ). Finally a pharmacological approach using 2-bromopalmitate $(\mathrm{BrP})$, a palmitate analog known to inhibit palmitoylation, leads to a similar lower Fas expression level (Figure $3 \mathrm{c}$ and Supplementary Figures S3d and e). Altogether, these results show that the DHHC7-mediated palmitoylation of Fas increases both total and plasma membrane Fas expression.

We previously demonstrated that Fas localization within membranes in nanodomains enriched in cholesterol and glycosphingolipids is crucial for Fas capacity to induce cell death, and that Fas palmitoylation is one signal allowing this constitutive association. ${ }^{8,17}$ Biochemically, these nanodomains can be isolated from cell lysate thanks to their insolubility in non-ionic detergent, and known as detergentresistant membrane nanodomains (DRMs). We subjected SW480 Fas WT cells to DRM isolation and showed that $27 \%$ of Fas is located in the DRM fractions, whereas only $14 \%$ when $\mathrm{DHHC7}$ is knocked down (Figure 3d), validating by a genetic approach that $\mathrm{DHHC} 7$ is allowing Fas to be targeted to specific membrane nanodomains probably by palmitoylation. Finally, decreasing $\mathrm{DHHC7}$ expression also reduces Fas organization in a higher-molecular weight complex, which was already described as a palmitoylation-dependent event (Figure 3e). ${ }^{9}$

Thus, DHHC7-mediated Fas palmitoylation not only regulates the Fas expression level but also repartition and organization in membranes.

Palmitoylation regulates Fas stability. As palmitoylation has been described to regulate the stability of several transmembrane proteins, ${ }^{11,26,27}$ we wish to compare the stability of WT and palmitoylation-deficient mutant Fas proteins and treated SW480 Fas WT and SW480 Fas C199V cells with cycloheximide $(\mathrm{CHX})$ to block protein neosynthesis. Whereas Fas WT is still detected after $16 \mathrm{~h}$ of $\mathrm{CHX}$ treatment, a decrease of Fas C199V expression can be observed upon $8 \mathrm{~h}$ of $\mathrm{CHX}$ exposure demonstrating that a palmitoylation-deficient $\mathrm{Fas}$ is less stable than its WT counterpart (Figure 4a). In order to assess whether this loss of Fas stability occurs at the plasma membrane, cell surface proteins of SW480 Fas WT and Fas C199V were biotinylated and cells cultured for different times to chase the biotinylated proteins. We first confirmed that Fas C199V is 
a
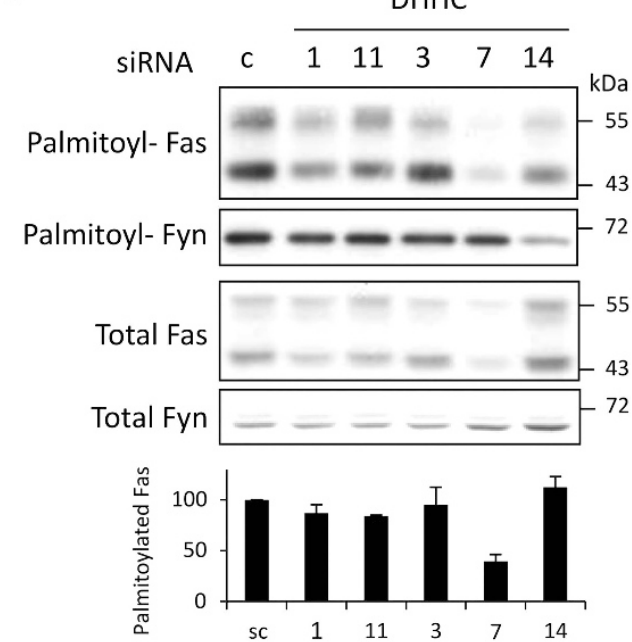

c

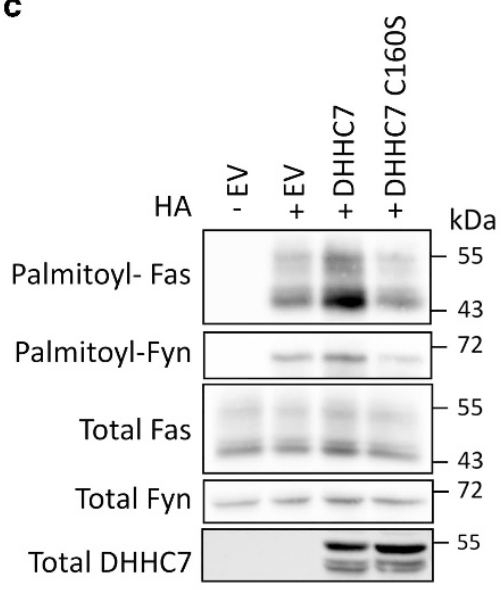

e

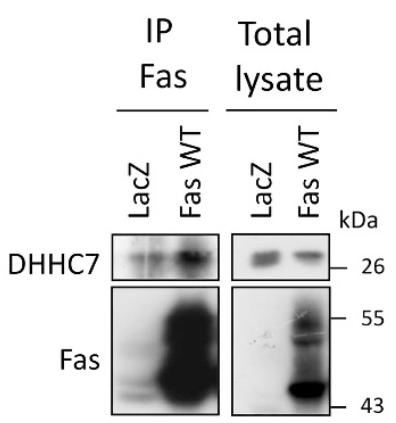

b

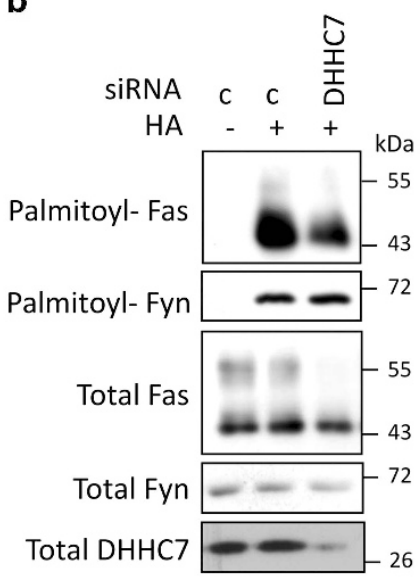

d

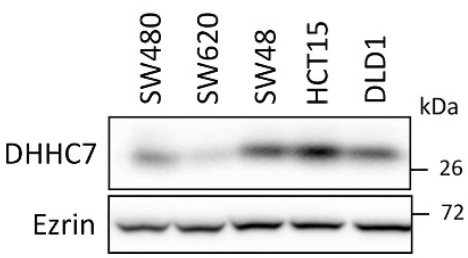

$\mathbf{f}$
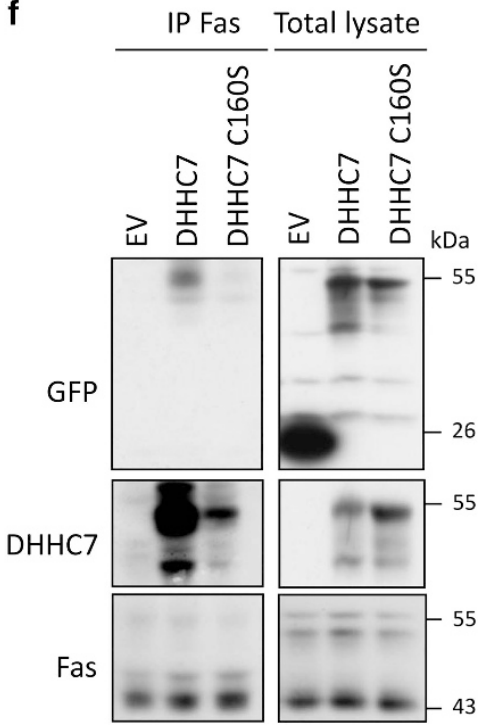

Figure 2 DHHC7 is a Fas-palmitoylating enzyme. (a) HEK293 cells were transfected for $48 \mathrm{~h}$ with $10 \mathrm{nM}$ siRNA control (scramble) or targeting the indicated DHHC (2 siRNA/DHHC) and submitted to ABE to monitor Fas palmitoylation status. The palmitoylated Fas was quantified by dividing the relative palmitoylated Fas (palmitoylated Fas/total Fas) by palmitoylated Fyn upon western blot quantification of three independent experiments. (b and c) SW480 transfected for $48 \mathrm{~h}$ with $10 \mathrm{nM}$ of siRNA control or siRNA targeting DHHC7 (b) or transfected with vectors coding for AcGFP alone (EV), or the fusion proteins DHHC7-AcGFP and DHHC7 C160S-AcGFP (c) were lysed and submitted to ABE to assess Fas palmitoylation status. Total DHHC7 in c shows the DHHC-AcGFP transfected level. (d) DHHC7 expression level was determined by immunoblotting of total lysate from indicated CRC cell lines after SDS-PAGE. (e) Lysates from SW480 LacZ and SW480 Fas WT cells was incubated with anti-Fas antibody (Ap01.3) for immunoprecipitation of endogenous and overexpressed Fas, respectively. Upon SDS-PAGE, Fas and the co-immunoprecipitated DHHC7 were immunoblotted. (f) SW480 were transiently transfected with vectors coding for AcGFP alone (EV), or the fusion proteins DHHC7-AcGFP and DHHC7 C160S-AcGFP and submitted to endogenous Fas immunoprecipitation. After SDS-PAGE, the co-immunoprecipitation of DHHC7-cGFP and DHHC7 C160S-AcGFP was detected by immunoblotting with anti-DHHC and anti-GFP antibody 


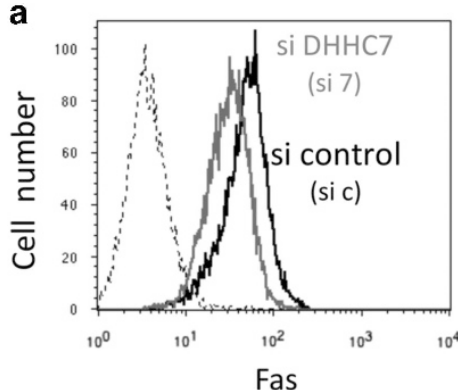

b

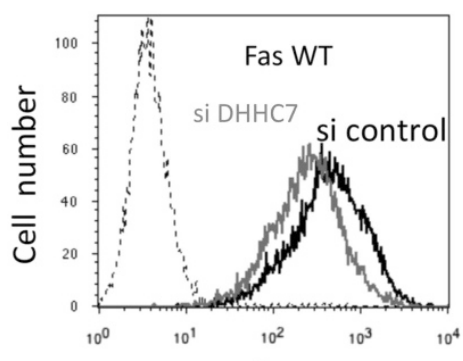

Fas

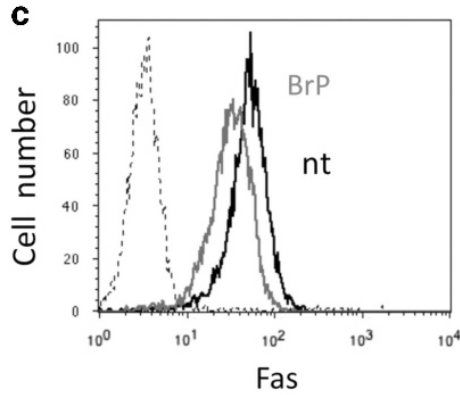

d

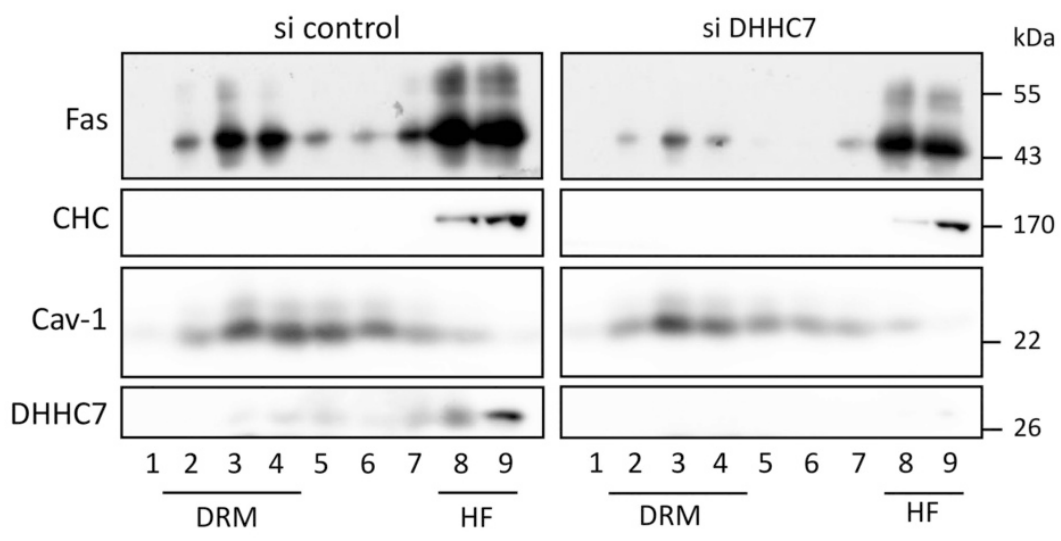

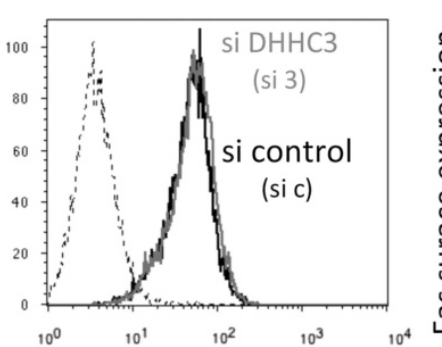

Fas
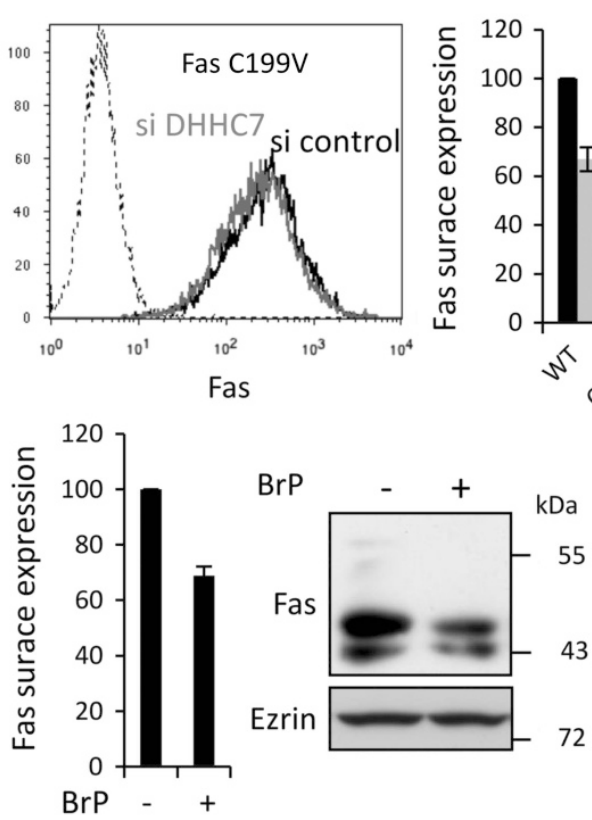
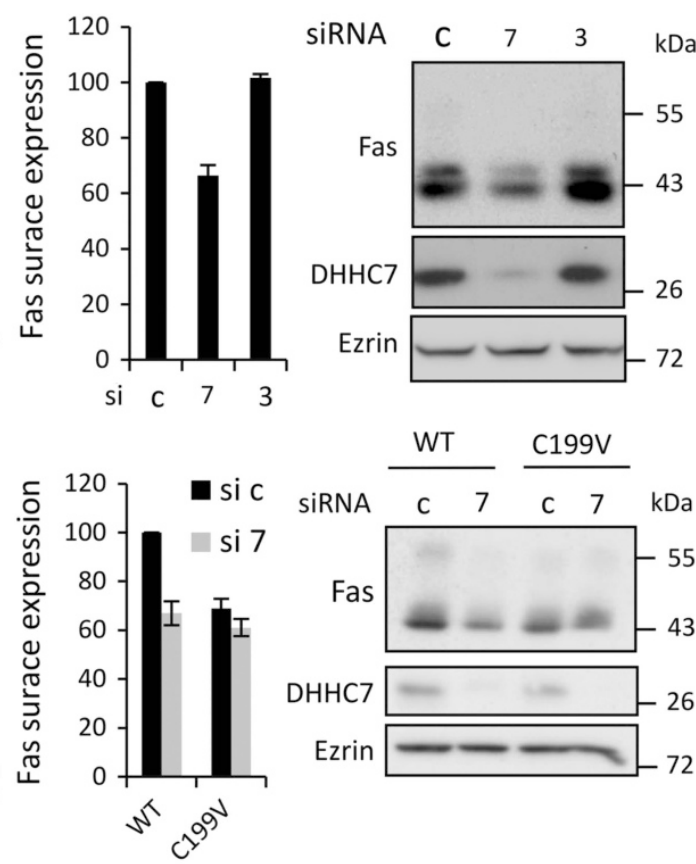

e

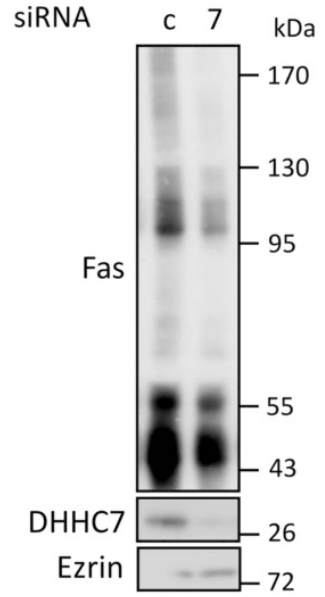

Figure 3 DHHC7-mediated palmitoylation regulates Fas expression and organization in membranes. (a and b) SW480 cells (a) or SW480 Fas WTand SW480 Fas C199V (b) transfected for $48 \mathrm{~h}$ with $10 \mathrm{nM}$ of the indicated siRNA were analyzed by flow cytometry for Fas surface expression (left panel), or lysed and submitted to SDS-PAGE for immunoblotting detection of total Fas expression (right panel). Quantification of Fas surface expression by flow cytometry represents the median of DX2-PE fluorescence (mean of 4 experiments \pm S.D.). (c) SW480 were exposed for $16 \mathrm{~h}$ to $100 \mathrm{nM} \mathrm{BrP}$ and analyzed by flow cytometry for Fas surface expression (left panel) or lysed and submitted to SDSPAGE for immunoblotting detection of total Fas expression (right panel). Quantification of flow cytometry data represents the median of DX2-PE fluorescence (mean of four experiments \pm S.D.). (d) Analysis of Fas partitioning in DRM was conducted on SW480 cells transfected for $48 \mathrm{~h}$ with $10 \mathrm{nM}$ with siRNA control or targeting DHHC7 upon Brij98 solubilization. Sucrose gradient fractions are immunoblotted with the indicated antibodies, Fyn and clathrin heavy chain (CHC) being used as DRM (fractions 1-4) and heavy fractions (8-9) markers, respectively. (e) SW480 Fas WT cells transfected for $48 \mathrm{~h}$ with $10 \mathrm{nM}$ with siRNA control or targeting DHHC7 were solubilized with Brij98 and resolved by SDS-PAGE in non-reducing conditions in order to detect monomers and oligomers of Fas by immunoblotting 
a

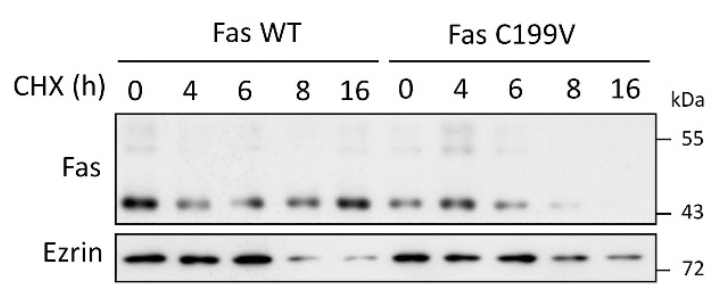

b
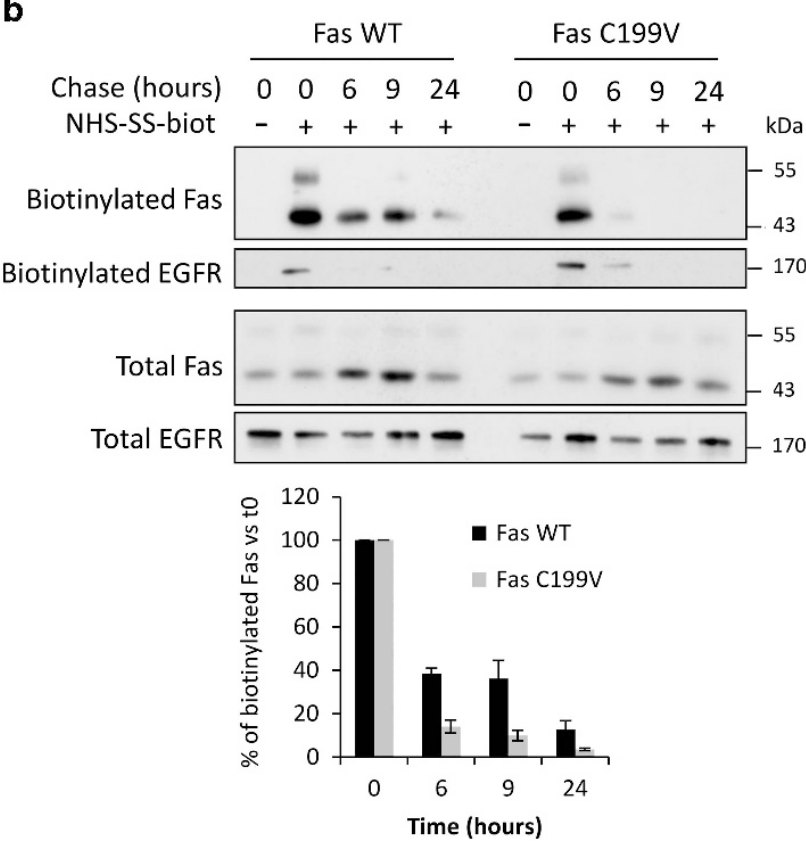

Figure 4 Palmitoylation of the Fas receptor regulates its stability. (a) Total lysates from SW480 Fas WT and SW480 Fas C199V exposed to $100 \mu \mathrm{g} / \mathrm{ml}$ of the protein synthesis inhibitor cycloheximide (CHX) for 4, 6, 8 and $16 \mathrm{~h}$ were resolved by SDSPAGE and immunoblotted with anti-Fas and anti-Ezrin antibodies. (b) A protein surface biotinylation protocol was performed on SW480 Fas WTand Fas C199V cells. Following the biotinylation step, cells were cultured for the indicated time, lysed, and cell lysate was submitted to streptavidin immunoprecipitation, SDS-PAGE and immunoblotting. Fas expressed at cell surface and biotinylated at $t=0$ was detected by anti-Fas antibody and EGFR was used as an internal control for equivalent protein surface biotinylation. Quantification of biotinylated Fas represented at the bottom of the figure is a mean of three independent experiments

less expressed in total and at the cell surface, and is more rapidly degraded from cell surface localization (Figure 4b). Therefore, Fas palmitoylation stabilizes Fas, notably at the plasma membrane.

Fas palmitoylation protects Fas from lysosomal degradation. Integral membrane proteins are generally targeted to lysosomes for degradation. Indeed, inhibiting the lysosomal pathway by chloroquine or bafilomycin $A 1$, which prevents acidification of endosomes or leupeptin that inhibits lysosomal proteases, induces an accumulation of Fas (Figure 5a). In order to decipher the role of palmitoylation in lysosomal targeting, SW480 Fas WT and Fas C199V cells were treated with increasing doses of lysosomal inhibitors. Fas WT only accumulates at the highest chloroquine dose, whereas low doses of both inhibitors restore Fas C199V expression at the level of the WT, suggesting that the palmitoylation-deficient mutant is more prone to lysosomal degradation and explains its lower stability (Figure 5b). Importantly, low doses of both chloroquine and bafilomycin $\mathrm{A} 1$ also restore Fas expression level in siRNA targeting DHHC7-transfected cells (Figure $5 \mathrm{c}$ ), thus confirming by inhibiting the endogenous Fas palmitoylation that palmitoylation protects Fas from lysosomal degradation.

We then looked whether Fas could be detected in lysosomes. Immunofluorescence analysis shows that some Fas WT-AcGFP colocalizes with the lysosome marker Lamp1 , whereas acGFP alone is excluded from lysosomal vesicles (Figure 5d). Moreover, colocalization seems to be even stronger when Fas C199V-AcGFP is expressed (Figure 5d). We biochemically isolated lysosomes from SW480 Fas WT and Fas C199V cells treated or not with chloroquine. Despite a lower expression of Fas C199V compared with Fas WT in total lysate, the palmitoylation-deficient mutant detection is stronger than its WT counterpart in the lysosomal fraction (Figure 5e). This difference is even enhanced by the chloroquine treatment (Figure 5e). Interestingly, when Fas was immunoprecipitated from Fas WT and Fas C199V cell lysate, Lamp-1 could be co-immunoprecipitated with Fas WT and even more strongly with Fas C199V (Figure 5f). Altogether, these results show that Fas is constitutively targeted to lysosomes for degradation, and that palmitoylation is a brake to this process.

Modulating DHHC7 level regulates Fas expression and Fas sensitivity to cell death in CRC cells. Decreasing Fas receptor expression level at the cell surface can be a way for cells to escape from FasL-induced cell death, particularly in a cancer context. We co-cultured SW480 target cells and the lymphoblastic Reh killer cells stably expressing Fas ligand (FasL) on their surface. Whereas SW480 cells die proportionally with the target/killer ratio, knocking down DHHC7 expression, which induces a significant decrease in Fas surface expression (Figure $3 \mathrm{a}$ and Supplementary Figure S3c), decreases the sensitivity of SW480 cells to membranebound FasL killing (Figure 6a). A time- and dose-dependent decrease in Fas-induced cell death was also detected when SW480 cells transfected with si DHHC7 were exposed to soluble FasL (Figure 6b).

If decreasing $\mathrm{DHHC7}$ level reduces Fas expression, the overexpression of DHHC7 could increase it. The SW620 CRC cell line, which originates from the same individual than SW480 but at a later stage of the disease, expresses less DHHC7 than SW480 and a very low level of Fas. Indeed, reinforcing $\mathrm{DHHC7}$ expression in SW620 by transfection can enhance total Fas expression dose dependently and only when $\mathrm{DHHC7}$ is catalytically active (Figure 6c). Importantly, this DHHC7-mediated increase of Fas expression can also be detected on the cell surface by a protein cell-surface biotinylation assay (Figure $6 \mathrm{~d}$ and Supplementary Figure S4) and is sufficient to sensitize the SW620 cells to FasLmediated cell death. As shown in Figure 6e, FasL hardly activates caspases 3 and 8 cleavage in control conditions, whereas cleaved caspases are detected in DHHC7expressing cells upon FasL exposure. Altogether, our results show that the expression level of $\mathrm{DHHC7}$, through its 


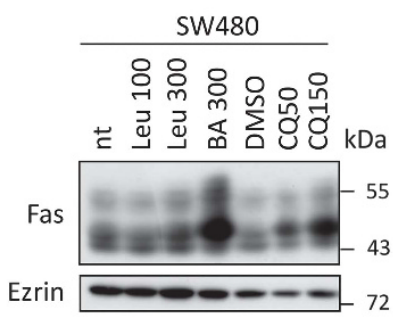

C

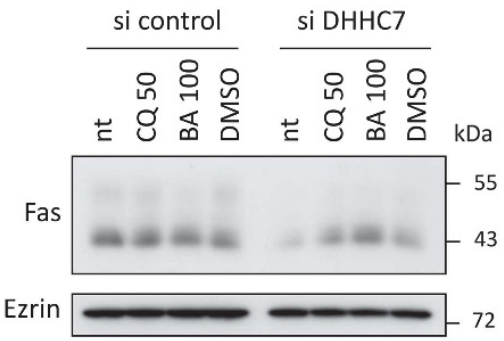

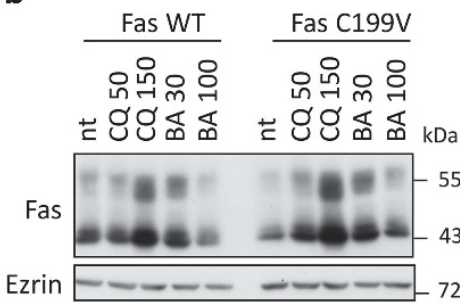

d
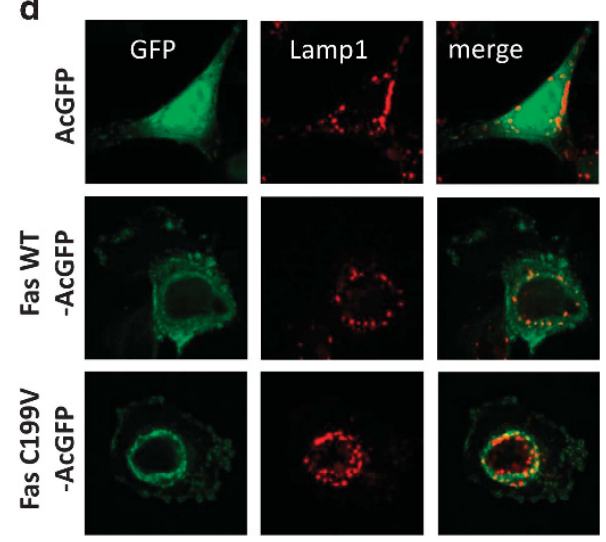

f e

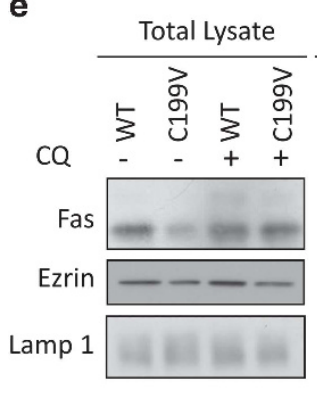

Lysosome fraction

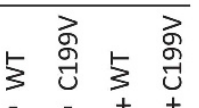

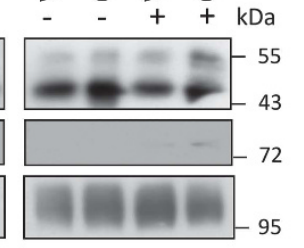

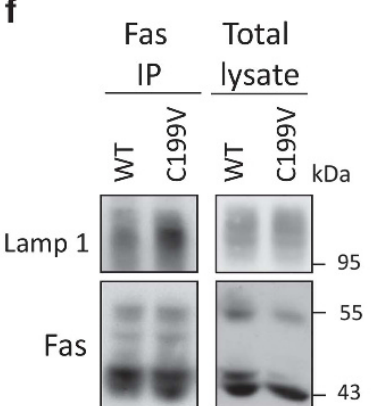

Figure 5 Palmitoylation protects the Fas receptor from lysosomal degradation. (a) The lysosomal degradation pathway inhibitors Leupeptin (100 and $300 \mu \mathrm{M})$, chloroquine $(50$ and $100 \mu \mathrm{M})$ and bafilomycin A1 (30, 100 and $300 \mathrm{nM}$ ) were added to SW480 (a), SW480 Fas WT and SW480 Fas C199V (b) cell medium for $16 \mathrm{~h}$ before cell lysis, SDSPAGE and immunoblotting with indicated antibodies. (c) SW480 transfected for $48 \mathrm{~h}$ with $10 \mathrm{nM}$ siRNA control or targeting DHHC7 were incubated for $16 \mathrm{~h}$ with $50 \mu \mathrm{M}$ chloroquine or $100 \mathrm{nM}$ bafilomycin A1 before lysis, SDS-PAGE and immunoblotting with indicated antibodies. (d) SW480 cells transfected with vectors coding for AcGFP, Fas WT-AcGFP and Fas C199V-AcGFP were immunostained with Lamp-1 antibody before confocal analysis. Images are representative of cells typically observed in the indicated conditions. (e) Isolation of lysosomal fraction was performed on SW480 Fas WT and SW480 Fas C199V cells with or without prior $16 \mathrm{~h}$ incubation with $50 \mu \mathrm{M}$ chloroquine. Total lysate before isolation and lysosomal fraction were resolved on SDS-PAGE followed by immunoblotting with indicated antibodies. V5 antibody was used to detect V5-tagged Fas, and Lamp-1 was used as a marker of lysosomes. (f) Fas immunoprecipitation was achieved on SW480 Fas WT and SW480 Fas C199V cell lysate. After SDS-PAGE, co-immunoprecipitation of Lamp-1 was detected by immunoblotting and V5-tagged Fas immunoprecipitation was verified with immunoblotting with V5 antibody after SDS-PAGE

enzymatic activity, clearly modulates Fas expression and therefore sensitivity to Fas-mediated cell death.

\section{Discussion}

We already reported the critical contribution of Fas palmitoylation to Fas localization in cholesterol and sphingolipidenriched nanodomains where it can efficiently transmit the death signal. ${ }^{8}$ We show here for the first time that Fas palmitoylation also occurs in epithelial cells from colorectal origin where it shows a pro-death role. By identifying here $\mathrm{DHHC7}$ as the main Fas palmitoyl acyltransferase and demonstrating that $\mathrm{DHHC7-mediated} \mathrm{palmitoylation} \mathrm{protects}$ Fas from lysosomal degradation, thereby regulating its expression, we decipher an additional role for Fas palmitoylation.

With the aim of better understanding the mechanisms regulating Fas palmitoylation, we screened the known DHHC palmitoyl acyltransferase family members to identify the enzyme(s) responsible for Fas palmitoylation. To do so, we applied a strategy (overexpression then siRNA) set up by Fukata et al. ${ }^{14,28,29}$ and used by several for the identification of $\mathrm{DHHC} /$ substrate pairs. Among the $23 \mathrm{DHHC}$ proteins, overexpression of 5 of them, namely $\mathrm{DHHC1} 111,3,7$ and 14 is able to enhance Fas palmitoylation. However, some studies suggested that overexpression may overcome the selectivity of $\mathrm{DHHC}$ protein. ${ }^{25}$ Indeed, when decreasing the endogenous level of these five preselected $\mathrm{DHHC}$ 

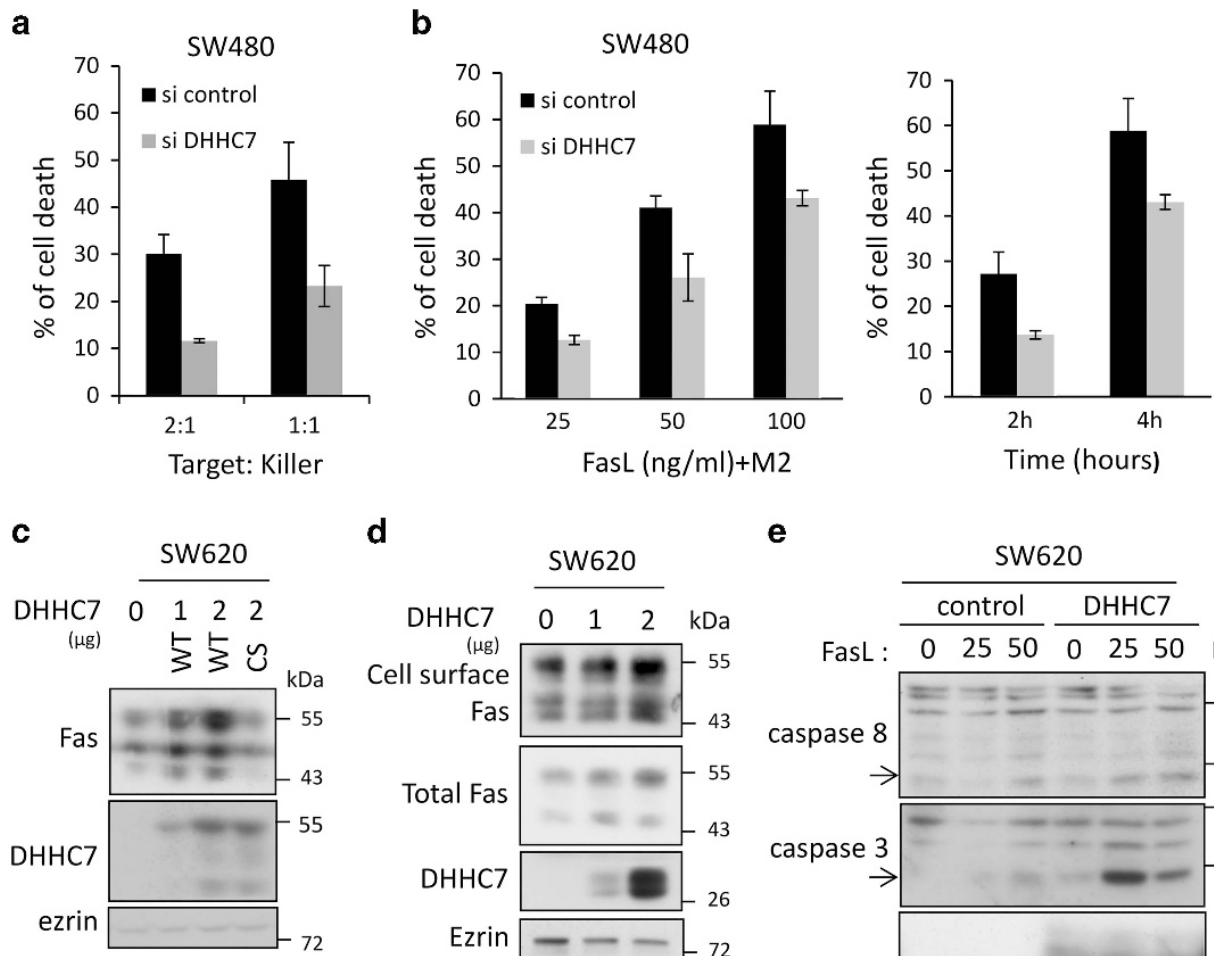

d

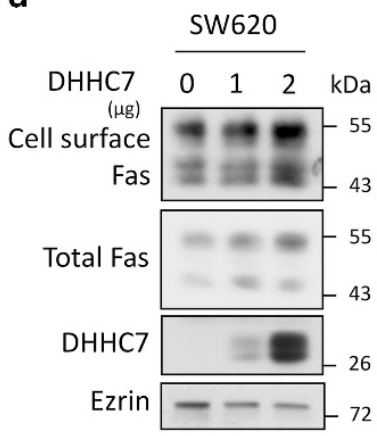

e

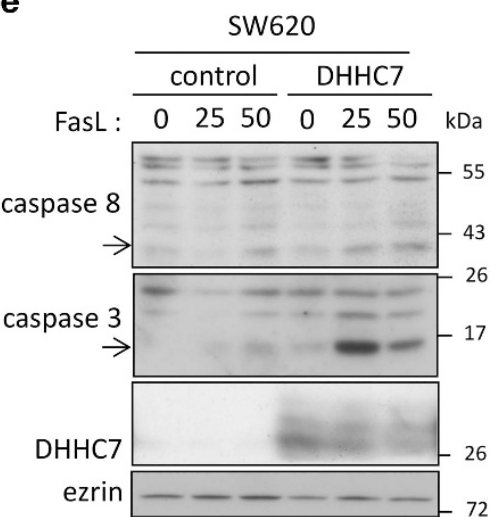

Figure 6 The modulation of the DHHC7 level regulates Fas expression and Fas sensitivity to cell death in CRC cells. (a) SW480 transfected for $48 \mathrm{~h}$ with $10 \mathrm{nM}$ siRNA control or targeting DHHC7 were then co-cultured for $24 \mathrm{~h}$ with Reh killer cells stably expressing FasL and monitored for cell death. Flow cytometry data represent mean \pm S.D. (b) Dose dependence (left panel) and kinetics (right panel) of Fas-induced cell death in SW480 cells transfected with siRNA control or targeting DHHC7. The dose dependence was investigated at $4 \mathrm{~h}$ of rhFasL+M2 exposure, and the cells used for the kinetics experiments were exposed to $100 \mathrm{ng} / \mathrm{ml} \mathrm{FasL} \mathrm{crosslinked} \mathrm{with} \mathrm{M} 2$ for the indicated time. Flow cytometry data represent mean \pm S.D. (c) Lysates from SW620 cells transfected with 1 or $2 \mu \mathrm{g}$ of plasmid coding for DHHC7-AcGFP (WT) or with $2 \mu \mathrm{g}$ of plasmid coding for DHHC7 C160S-AcGFP (CS) was resolved by SDS-PAGE and analyzed by immunoblotting. (d) Protein surface biotinylation assay to assess Fas expression at cell surface was carried out on SW620 cells transfected or not for $48 \mathrm{~h}$ with 1 or $2 \mu \mathrm{g}$ of plasmid coding for DHHC7-HA and analyzed by immunoblotting with indicated antibodies. (e) SW620 cells transfected for $48 \mathrm{~h}$ with DHHC7-HA or with empty vector as control were exposed to 25 and $50 \mathrm{ng} / \mathrm{ml}$ rhFasL crosslinked with M2 for $6 \mathrm{~h}$ before lysis, SDS-PAGE and immunoblotting with indicated antibodies. The cleaved form of caspase 3 (p17) and caspase 8 (p41/43) representing caspase activation are indicated with an arrow

candidates with siRNA, only the knockdown of DHHC7 significantly affects Fas palmitoylation, suggesting that $\mathrm{DHHC7}$ is the major enzyme that palmitoylates Fas in the tested cells.

The redundancy and specificity of DHHC enzymes is still a matter of debate. Whereas several studies concluded that particular DHHC(s) palmitoylate defined substrates, ${ }^{14,16,23,30}$ it was postulated that any Golgi-resident DHHC can palmitoylate the palmitoylable peripheral membrane proteins. ${ }^{31}$ Our results argue for some specificity of DHHC proteins toward their substrate, as only knockdown of $\mathrm{DHHC7}$ really impacts Fas palmitoylation. However, residual Fas palmitoylation is detected upon $\mathrm{DHHC7}$ silencing, which can be attributed to incomplete DHHC7 knockdown or to additional minor contribution from other $\mathrm{DHHC}$ proteins that the sensitivity of our assay did not allow to identify. Importantly, we could demonstrate a basal association between endogenous $\mathrm{DHHC7}$ and Fas, thus reinforcing the conclusion that $\mathrm{DHHC7}$ is a PAT for Fas. Indeed, a number of studies showed that palmitoylated proteins can assemble with their cognate $\mathrm{DHHC}$ during the palmitoylation process. ${ }^{16,23-25}$ We previously demonstrated that the TRAIL receptor DR4 is also palmitoylated, this palmitoylation contributing to DR4 ability to induce cell death by targeting DR4 to specific membrane nanodomains. ${ }^{32}$ Interestingly, a recent report links DHHC3, the closest DHHC member of DHHC7, as a DR4 interacting protein by a two-hybrid screen. ${ }^{33}$ Importantly, although the authors do not examine whether DHHC3 could indeed palmitoylate DR4, they demonstrate that $\mathrm{DHHC} 3$ is necessary for DR4 targeting to the plasma membrane, thus enhancing TRAIL sensitivity of the cells. The present study also shows a reduced expression of Fas at the plasma membrane upon $\mathrm{DHHC} 7$ silencing caused by increased instability of both plasma membrane and total nonpalmitoylated Fas. Thus, it seems that a similar molecular process, that is to say DHHC-mediated palmitoylation are controlling DR4 and Fas surface expression and thus death activity.

The concordance of results obtained with three complementary approaches (a genetic one where the palmitoylation site is mutated, the targeting of the palmitoylating enzyme and the pharmacological inhibition of palmitoylation) allows us to conclude that loss of palmitoylation downregulates Fas expression. Cysteines can be a site for alternative 
post-translational modifications, and Fas has been described to be nitrosylated in CRC cell lines on both cysteine 199 and $304 .{ }^{34}$ However, the nitrosylation was induced by a nitric oxide donor treatment but not detected in basal condition, and the pro-apoptotic functional effect of Fas nitrosylation was only demonstrated for cysteine 304. In our study, the use of two additional approaches lowers the possibility to involve another modification on C199.

Besides, the palmitoylation inhibition by bromopalmitate or the silencing of DHHC7 only slightly affects Fas C199V expression. Despite their lack of specificity on Fas targeting, these two approaches allow us to draw two types of conclusions: (i) the decrease of Fas expression obtained by silencing $\mathrm{DHHC} 7$ or with bromopalmitate mainly results from a direct effect on Fas palmitoylation itself; (ii) potential indirect contributions of additional bromopalmitate or $\mathrm{DHHC} 7$ targets can account for a minor part of the decrease of Fas expression, since the expression of the palmitoylationdeficient Fas is slightly reduced.

The basal difference in Fas expression between Fas WTand Fas C199V could not be observed in our initial work relating the role of Fas palmitoylation, ${ }^{8}$ since the comparison between Fas WT and Fas C199V was relying on cell clones chosen for their equivalent stable expression of both proteins. In contrast, in the present study, the experiments were conducted on an entire cell population obtained upon lentiviral infection. Altogether, our results prove that DHHC7-mediated Fas palmitoylation on cysteine 199 maintains an optimal Fas expression.

The reduction of Fas expression upon loss of Fas palmitoylation suggested that a palmitoylation-deficient Fas is more prone to instability. Indeed, Fas C199V, both total and plasma membrane expressed, show a decrease half-life compared with Fas WT, suggesting that Fas palmitoylation stabilizes Fas at the plasma membrane and prevents a premature degradation of the receptor. Indeed, palmitoylation of several transmembrane proteins of different functions such as integrin $a 6 \beta 4,{ }^{35} \mathrm{CCR} 5,{ }^{36}$ tetraspanin CD9 and CD151, ${ }^{27}$ A1 adenosine receptor, ${ }^{37}$ the anthrax receptor TEM $8,{ }^{26}$ the snare $\operatorname{Tlg} 1^{38}$ has been implicated in protein stability. The degradation pathway followed by integral proteins ends in lysosomes, which contains acidic proteases. Inhibition of these proteases restores the expression of palmitoylationdeficient CCR5, ${ }^{36}$ integrin $a 6 \beta 4,{ }^{35}$ tetraspanin CD9 and CD $151^{27}$ and TEM8. ${ }^{26}$ We demonstrate here the localization of Fas WT in lysosomes by both immunofluorescence and biochemical isolation, showing that Fas follows the lysosomal degradation pathway. Whether these Fas WT molecules represent only a non-palmitoylated population of Fas or a mixed pool containing also the palmitoylated one is still a question. However, one can notify that the detection of palmitoylation-deficient Fas in lysosomes is stronger in comparison with WT (see Figure 5e). Importantly, inhibiting the lysosomal degradation by chloroquine or bafilomycin A1 not only restores Fas C199V expression to the level of Fas WT but also Fas expression upon DHHC7 silencing to normal. Thus, we propose that Fas palmitoylation protects Fas for premature targeting to the lysosomal degradation pathway. The molecular mechanism involved in this lysosomal targeting is still not elucidated. However, some studies propose different scenarios in which palmitoylation prevents protein degradation by modifying these protein interactions with their lipidic or proteic environment within membranes. In the case of the SNARE Tlg1 in yeast, SWF1-mediated palmitoylation prevents Tlg1 association with the ubiquitin ligase Tul1, its further ubiquitination and targeting to multivesicular body where it is degraded. ${ }^{38}$ Palmitoylation of the anthrax receptor TEM8, by excluding TEM8 from raft nanodomains, segregates TEM8 from the ubiquitin ligase $\mathrm{cbl}$ and inhibits its ubiquitination, which is a prerequisite for endocytosis and targeting to lysosomes. ${ }^{26}$ In the case of the tetraspanins CD9 and CD151, palmitoylation promotes homo- and hetero-clustering of tetraspanins into multiprotein complexes known as the tetraspaninenriched microdomain. This TEM localization might participate in palmitoylation-mediated tetraspanin stabilization. ${ }^{27}$ Fas palmitoylation by $\mathrm{DHHC7}$ is targeting Fas in raft nanodomains and promotes its oligomerization. We can hypothesize that Fas is locked in multiprotein complexes within lipid rafts by palmitoylation and that non-palmitoylated Fas being excluded from these complexes can follow the degradation pathway. The involvement of an ubiquitination step to regulate Fas lysosomal targeting in this context is not excluded.

Fas palmitoylation contributes to an optimal Fas-mediated cell death by targeting Fas to the raft nanodomains. But we additionally demonstrate here that Fas palmitoylation also promotes Fas-induced cell death by maintaining a proper Fas expression level. Therefore, we show that modulating DHHC7 level, by RNAi or overexpression, directly affects Fas expression and notably at the plasma membrane, which influences cellular sensitivity to Fas-mediated cell death. Cancer cells have developed mechanisms to evade from cell death and among them, downregulating Fas expression. ${ }^{39,40}$ Interestingly, an expression profiling analysis describes a decrease in $\mathrm{DHHC7}$ mRNA level during intestinal APC-driven tumorigenesis by analyzing polyps of both hereditary CRC patients and mice carrying an inactivating APC mutation. ${ }^{41}$ Indeed, if we compare the CRC cells model SW480/SW620, which originates from the same patients but at different stages of the disease, SW480 coming from the primary tumor and SW620 one year later from a lymph node, the later shows a decrease in both $\mathrm{DHHC7}$ and Fas expression compared with SW480. Therefore, it can be postulated that a decrease in $\mathrm{DHHC7}$ expression during colorectal tumorigenesis can decrease Fas expression by affecting Fas palmitoylation and then allowing cancer cells to become more resistant to Fas-induced cell death.

\section{Materials and Methods}

Antibodies. Fas antibody used for immunoprecipitation (Apo1.3) is from Alexis (San Diego, CA, USA) and those used for immunoblotting are from Epitomics (EPR520 and EPR5700; Burlingame, CA, USA) and from Santa Cruz Biotechnology (B10; Dallas, TX, USA). An antibody directed against the V5 tag (Invitrogen, Carlsbad, CA, USA) was also used to detect Fas-V5. For immunoblotting and immunofluorescence, anti-Lamp-1 antibody comes from Cell Signaling Technology (Danvers, MA, USA). For immunoblotting, anti-Fyn, anti-CHC and anti-caveolin1 are from Santa Cruz Biotechnology, anti-EGFR from Cell Signaling Technology, anti-Caspase 8 from MBL (Woburn, MA, USA), anti-caspase 3 from Becton Dickinson (Franklin Lakes, NJ, USA), anti-GFP from Abcam (Cambridge, UK), anti-DHHC7 from Novus Biologicals (Littleton, CO, USA) and anti-Ezrin from Zymed (San Francisco, CA, USA). For FasL treatment, Flag-recombinant human FasL (rhFasL) from Alexis is crosslinked with anti-Flag M2 from Sigma (St Louis, MO, USA). 
Constructs. Lentiviral constructs pLenti6-hFasWT-V5, pLenti6-hFasC199V-V5, pLenti6-hFasWT-AcGFP, pLenti6- hFasC199V-AcGFP were obtained by subcloning from pcR3 hFasWT and pcR3 hFasC199V. ${ }^{8}$

Individual DHHC clone HA-tagged or EGFP-tagged was a gift from M. Fukata. ${ }^{28}$ DHHC7 was subcloned in plenti6-AcGFP and plenti6-DHHC7 C160S-AcGFP was obtained by site-directed mutagenesis.

pEYFP-Golgi from Clontech (Mountain View, CA, USA) was used as a Golgi marker in immunofluorescence. It encodes a fusion protein consisting of enhanced yellow fluorescent protein (EYFP) and a sequence encoding the N-terminal 81 amino acids of human beta 1,4-galactosyltransferase that targets the fusion protein to the trans-medial region of the Golgi apparatus.

Cell culture, treatment and transfection. SW480, SW620, SW48, DLD1, HCT15 and Reh pcR33FasL were grown in RPMI supplemented with $10 \%$ fetal calf serum and HEK293 in DMEM supplemented with $10 \%$ fetal calf serum.

SW480 LacZ, SW480 Fas WT and SW480 Fas C199V were obtained by transduction of the corresponding lentiviral construct in SW480 cells followed by blasticidin (Invitrogen) selection. To do so, lentivirus stock were produced by cotransfection of HEK293 cells with the pseudotyping vector pMD2.G, the packaging vector p8.91 and the lentiviral cloning vector plenti6 encoding the protein of interest.

Transient transfection of plasmids and siRNA was obtained by using the Jetprime transfection reagent (Polyplus transfection) or the Interferin transfection reagent (Polyplus, Illkirch-Graffenstaden, France), respectively. SiRNA used to target the different DHHC proteins or siRNA scramble used as a control come from Qiagen (Germantown, MD, USA).

For palmitoylation inhibition, cells were grown for $16 \mathrm{~h}$ in $0.1 \%$ bovine serum albumin fatty acid-free in presence of $100 \mathrm{mM}$ 2-bromopalmitate (Fluka, Buchs, Switzerland). In order to inhibit the lysosomal degradation pathway, chloroquine (Sigma), bafilomycine A1 (Calbiochem, Gibbstown, NJ, USA) or leupeptin (Calbiochem) were added to the culture medium for $16 \mathrm{~h}$. Protein synthesis inhibition was studied by adding cycloheximide ( $100 \mu \mathrm{g} / \mathrm{ml}$; Sigma) to the culture medium.

Cell death assay. For cell death experiments, cells $\left(2.5 \times 10^{5}\right)$ were incubated with indicated doses of recombinant human Flag-FasL (rhFasL) crosslinked with $1 \mu \mathrm{g} / \mathrm{ml} \mathrm{M} 2$ antibody in culture medium at $37^{\circ} \mathrm{C}$ and $5 \% \mathrm{CO}_{2}$. Alternatively, for cell death induced by membrane-bound FasL, target cells were co-cultured with FasLexpressing Reh cells (killer) or with FasL-negative Reh cells (control). Killer and control cells were prestained with $1 \mu \mathrm{M}$ diacetate succinimidyl ester (CFSE) prior to co-culture to distinguish them from target cells during subsequent flow cytometry analysis.

After the indicated time, cells were collected and fixed in ice-cold $70 \%$ ethanol.

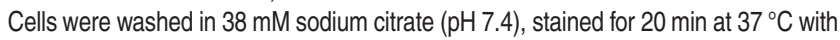
$50 \mu \mathrm{g} / \mathrm{ml}$ propidium iodide (Sigma) and $5 \mu \mathrm{g} / \mathrm{ml}$ RNaseA (Sigma) in $38 \mathrm{mM}$ sodium citrate and analyzed by flow cytometry using the Fortessa analyser (Becton Dickinson). The proportion of apoptotic cells as represented by the sub-G1 peak was determined.

Colony formation assay. Two hundred and fifty SW480 Fas WT or SW480 Fas C199V cells were plated in a six-well plate. The following day (after cell adhesion), the indicated rhFasL treatment was added to culture media. After 10 days incubation, cells were fixed with ethanol and colored with crystal violet to visualize the colonies. The number of colonies of a minimum determined size was quantified after scanning using the Image $\mathrm{J}$ software.

Immunoprecipitation and western blotting. Cells were lysed with buffer A (25 mM Hepes, $150 \mathrm{mM} \mathrm{NaCl}, 1 \mathrm{mM}$ EGTA, $10 \mathrm{mM} \mathrm{NaF}, 10 \mathrm{mM}$ Napyrophosphate, $5 \mathrm{mM}$ sodium orthovanadate, protease inhibitors cocktail) containing $1 \% \mathrm{NP}-40$ and $10 \%$ glycerol (lysis buffer) at $4{ }^{\circ} \mathrm{C}$. The post-nuclear supernatants obtained after centrifugation at $800 \times \mathrm{g}$ for $10 \mathrm{~min}$ at $4^{\circ} \mathrm{C}$ were incubated with the anti-Fas antibody Apo1.3 (Alexis) coupled to protein G-sepharose beads (Zymed) overnight. After four washes with lysis buffer, beads were eluted with Laemmli buffer at $95^{\circ} \mathrm{C}$ for $5 \mathrm{~min}$ and subjected to SDS-polyacrylamide gel electrophoresis (SDSPAGE), followed by transfer to a PVDF membrane (Immobilon-P; Millipore, Molsheim, France) and immunoblotting with the indicated primary antibodies.

Western blot analysis of total proteins was performed according to standard protocols. Briefly, cells were lysed with Laemmli buffer. After sonication, quantification, denaturing and reduction (dithiothreitol $1 \mathrm{mM}, 95^{\circ} \mathrm{C}, 5 \mathrm{~min}$ ) the solubilised proteins were resolved by SDS-PAGE. When non-reducing conditions were necessary, dithiothreitol and $95^{\circ} \mathrm{C}$ heating were omitted. Immunoblotting was performed using indicated primary antibody and HRP-coupled secondary antibody (Jackson ImmunoResearch, West Grove, PA, USA). For the V5 antibody, in case of low signal, an amplification step using a biotin-linked secondary antibody and streptavidin-HRP was also used.

Flow cytometry analysis of Fas surface expression. SW480 cells were collected and incubated on ice with anti-Fas antibody DX2 conjugated with phycoerythrin (Miltenyi Biotech, Bergish Gladbach, Germany) for 30 min or with anti TNFR1 (R\&D systems, Minneapolis, MN, USA) following with FITC-coupled antimouse antibody. After two washing steps, cells were resuspended in cold PBS and analyzed on the FACScalibur or Fortessa cytometers (Becton Dickinson).

Protein surface biotinylation. The whole biotinylation procedure is performed imperatively on ice with cold buffer to prevent protein surface internalization. Adherent cells on a six-well plate were washed three times with biotinlylation buffer (PBS pH 8.0, $1 \mathrm{mM} \mathrm{Mgcl2,0.5} \mathrm{mM} \mathrm{CaCl2),} \mathrm{incubated} \mathrm{with} 1 \mathrm{ml}$ biotinylation buffer $+1 \mathrm{mg} / \mathrm{ml}$ NHS-SS-Biotin (Pierce, Rockford, IL, USA) for $20 \mathrm{~min}$, washed once and incubated for 10 min with quenching solution (biotinylation buffer $+100 \mathrm{mM}$ glycine) and washed three times with biotinylation buffer. Upon this biotinylation step, cells were either put back in culture for the indicated time before lysis or directly lysed with $1 \%$ SDS buffer. After a sonication step, lysate was diluted to decrease SDS concentration and submitted to immunoprecipitation with neutravidin beads (Pierce). After SDS-PAGE resolution, biotinylated proteins were detected by immunoblotting.

Protein palmitoylation detection by ABE technic. Protein palmitoylation was detected by a previously described method adapted from the acyl-biotinyl exchange (ABE) protocol of Drisdel and Green. ${ }^{42}$ Briefly, $1.5 \mathrm{mg}$ of protein lysate (250 mM HEPES, $1 \mathrm{mM}$ EDTA and $2.5 \%$ SDS) were incubated with $20 \mathrm{mM}$ methyl methanethiosulfonate (Sigma) at $50^{\circ} \mathrm{C}$ for $20 \mathrm{~min}$ to block free thiols. Proteins were precipitated with acetone and resuspended in $1 \mathrm{M}$ hydroxylamine pH 7.4 (Sigma) that specifically release thioesther-linked palmitoyl moieties and restore the modified cysteines to thiols, which were then mixed with $0.2 \mathrm{mM}$ biotin-HPDP (Pierce) for $1 \mathrm{~h}$ at room temperature. Biotinylated proteins were purified with neutravidin beads (Pierce), separated by SDS-PAGE and submitted to immunoblotting with antibody against the protein of interest. Specificity of the experiment is controlled by omitting the hydroxylamine treatment.

DRM preparation. Briefly, post-nuclear supernatant from SW480 cells $\left(10 \times 10^{6}\right)$ were solubilised in $1 \mathrm{ml}$ of Buffer A with $1 \%$ Brijg8 (Sigma) for $1 \mathrm{~h}$ at $4{ }^{\circ} \mathrm{C}$ and diluted with $2 \mathrm{ml}$ buffer $A$ containing $2 \mathrm{M}$ sucrose, before being placed at the bottom of a step sucrose gradient (1.33-0.9-0.8-0.75-0.7-0.6-0.5-0.4-0.3-0.2 M) in buffer A. After 16-h centrifugation at $4{ }^{\circ} \mathrm{C}$ at 38000 r.p.m. in a SW41 rotor (Beckman Coulter, Fullerton, CA, USA), eight $1 \mathrm{ml}$ fractions and one $3 \mathrm{ml}$ fraction (fraction 9) were harvested from the top. An equal volume of each fraction was diluted 1:1 with Laemmli buffer, heated to $95^{\circ} \mathrm{C}$ and subjected to SDS-PAGE and immunoblotting.

Lysosome enrichment. SW480 Fas WT or SW480 Fas C199V cells pretreated or not with $50 \mathrm{mM}$ chloroquine for $16 \mathrm{~h}$ were subjected to a lysosomeenrichment protocol following the manufacturer's instructions (Thermo scientific, Waltham, MA, USA). Briefly, $20 \times 10^{6}$ cells were solubilized with indicated buffers and a sonication step. After protein quantification, lysate was separated on an Optiprep discontinuous density gradient (from 30 to $17 \%$ ) by $2 \mathrm{~h}$ ultracentrifugation at $145000 \times g$ at $4{ }^{\circ} \mathrm{C}$. Lysate before the isolation step and recovered fractions were mixed with laemmli buffer, separated on SDS-PAGE, and immunoblotted. Lamp-1 was used as a lysosome marker to verify the quality of lysosome enrichment.

Immunofluorescence. SW480 cells were allowed to adhere to coverslips for $24 \mathrm{~h}$ in a 24-well plate and transfected with the indicated plasmids. After $48 \mathrm{~h}$, cells were fixed with $4 \%$ paraformaldehyde, $0.2 \%$ glutaraldehyde, $3 \%$ sucrose for $30 \mathrm{~min}$ at room temperature and subsequently permeabilized with digitonin $10 \mu \mathrm{g} / \mathrm{ml}$ for $45 \mathrm{~min}$. Lamp-1 was detected with corresponding primary antibodies followed by Alexa Fluor 546 conjugated anti-rabbit secondary antibody (Molecular Probe, Eugene, OR, USA). Images were obtained using a laserscanning microscope (Olympus/Andor technology) with a $\times 100$ objective lens. All experimental and control images of the immunofluorescence data were collected using identical imaging settings. 


\section{Conflict of Interest}

The authors declare no conflict of interest.

Acknowledgements. We are grateful to Sébastien Huault and Agnès Loubat for support assistance and Biljana Hadzic for help in project initiation. We thank Masaki Fukata for kindly providing the DHHC constructs. This work was supported by the French Government (National Research Agency, ANR) through the "Investments for the Future" LABEX SIGNALIFE: program reference \# ANR-11-LABX-0028-01, institutional funds from the Centre National de la Recherche Scientifique, the Institut National de la Santé et de la Recherche Medicale and by grants from the Institut national du cancer and the Agence Nationale de la Recherche.

1. Ashkenazi A, Dixit VM. Death receptors: signaling and modulation. Science 1998; $\mathbf{2 8 1}$ 1305-1308.

2. Kischkel FC, Hellbardt S, Behrmann I, Germer M, Pawlita M, Krammer PH et al. Cytotoxicity-dependent APO-1 (Fas/CD95)-associated proteins form a death-inducing signaling complex (DISC) with the receptor. EMBO J 1995; 14: 5579-5588.

3. Peter ME, Budd RC, Desbarats J, Hedrick SM, Hueber AO, Newell MK et al. The CD95 receptor: apoptosis revisited. Cell 2007; 129: 447-450.

4. Martin-Villalba A, Llorens-Bobadilla E, Wollny D. CD95 in cancer: tool or target?. Trends Mo Med 2013; 19: 329-335.

5. Chen L, Park SM, Turner JR, Peter ME. Cell death in the colonic epithelium during inflammatory bowel diseases: CD95/Fas and beyond. Inflamm Bowel Dis 2010; 16 1071-1076.

6. Hoogwater FJ, Steller EJ, Westendorp BF, Borel Rinkes IH, Kranenburg O. CD95 signaling in colorectal cancer. Biochim Biophys Acta 2012; 1826: 189-198.

7. Zheng HX, Cai YD, Wang YD, Cui XB, Xie TT, Li WJ et al. Fas signaling promotes motility and metastasis through epithelial-mesenchymal transition in gastrointestinal cancer. Oncogene 2013; 32: 1183-1192.

8. Chakrabandhu K, Hérincs Z, Huault S, Dost B, Peng L, Conchonaud F et al. Palmitoylation is required for efficient Fas cell death signaling. EMBO J 2007; 26: 209-220.

9. Feig C, Tchikov V, Schütze S, Peter ME. Palmitoylation of CD95 facilitates formation of SDS-stable receptor aggregates that initiate apoptosis signaling. EMBO J 2007; 26: 221-231.

10. Guardiola-Serrano F, Rossin A, Cahuzac N, Lückerath K, Melzer I, Mailfert S et al. Palmitoylation of human FasL modulates its cell death-inducing function. Cell Death Dis 2010; 1: e88.

11. Linder ME, Deschenes RJ. Palmitoylation: policing protein stability and traffic. Nat Rev Mol Cell Biol 2007; 8: 74-84.

12. Iwanaga $T$, Tsutsumi R, Noritake J, Fukata $Y$, Fukata M. Dynamic protein palmitoylation in cellular signaling. Prog Lipid Res 2009; 48: 117-127.

13. Blaskovic S, Blanc M, van der Goot FG. What does S-palmitoylation do to membrane proteins?. FEBS J 2013; 280: 2766-2774.

14. Tsutsumi R, Fukata Y, Fukata M. Discovery of protein-palmitoylating enzymes. Pflugers Arch 2008; 456: 1199-1206.

15. Ohno Y, Kihara A, Sano T, Igarashi Y. Intracellular localization and tissue-specific distribution of human and yeast $\mathrm{DHHC}$ cysteine-rich domain-containing proteins. Biochim Biophys Acta 2006; 1761: 474-483.

16. Greaves J, Chamberlain LH. DHHC palmitoyl transferases: substrate interactions and (patho)physiology. Trends Biochem Sci 2011; 36: 245-253.

17. Hueber AO, Bernard AM, Herincs Z, Couzinet A, He HT. An essential role for membrane rafts in the initiation of Fas/CD95-triggered cell death in mouse thymocytes. EMBO Rep 2002; 3: 190-196.

18. Hueber AO. Role of membrane microdomain rafts in TNFR-mediated signal transduction Cell Death Differ 2003; 10: 7-9.

19. Henkler F, Behrle E, Dennehy KM, Wicovsky A, Peters N, Warnke C et al. The extracellular domains of FasL and Fas are sufficient for the formation of supramolecular FasL-Fas clusters of high stability. J Cell Biol 2005; 168: 1087-1098.
20. Miyaji M, Jin ZX, Yamaoka S, Amakawa R, Fukuhara S, Sato SB et al. Role of membrane sphingomyelin and ceramide in platform formation for Fas-mediated apoptosis. J Exp Med 2005; 202: 249-259.

21. Gajate C, Del Canto-Jañez E, Acuña AU, Amat-Guerri F, Geijo E, Santos-Beneit AM et al. Intracellular triggering of Fas aggregation and recruitment of apoptotic molecules into Fas-enriched rafts in selective tumor cell apoptosis. J Exp Med 2004; 200: 353-365.

22. Rossin A, Kral R, Lounnas N, Chakrabandhu K, Mailfert S, Marguet D et al. Identification of a lysine-rich region of Fas as a raft nanodomain targeting signal necessary for Fas-mediated cell death. Exp Cell Res 2010; 316: 1513-1522.

23. Lu D, Sun $H Q$, Wang $H$, Barylko B, Fukata $Y$, Fukata $M$ et al. Phosphatidylinositol 4-kinase $\| \alpha$ is palmitoylated by Golgi-localized palmitoyltransferases in cholesteroldependent manner. J Biol Chem 2012; 287: 21856-21865.

24. Tian L, McClafferty H, Jeffries $\mathrm{O}$, Shipston MJ. Multiple palmitoyltransferases are required for palmitoylation-dependent regulation of large conductance calcium- and voltage-activated potassium channels. J Biol Chem 2010; 285: 23954-23962.

25. Hou H, John Peter AT, Meiringer C, Subramanian K, Ungermann C. Analysis of DHHC acyltransferases implies overlapping substrate specificity and a two-step reaction mechanism. Traffic 2009; 10: 1061-1073.

26. Abrami L, Leppla SH, van der Goot FG. Receptor palmitoylation and ubiquitination regulate anthrax toxin endocytosis. J Cell Biol 2006; 172: 309-320.

27. Sharma $\mathrm{C}$, Yang $\mathrm{XH}$, Hemler ME. DHHC2 affects palmitoylation, stability, and functions of tetraspanins CD9 and CD151. Mol Biol Cell 2008; 19: 3415-3425.

28. Fukata M, Fukata Y, Adesnik H, Nicoll RA, Bredt DS. Identification of PSD-95 palmitoylating enzymes. Neuron 2004; 44: 987-996.

29. Tsutsumi R, Fukata $Y$, Noritake J, Iwanaga T, Perez F, Fukata M. Identification of G protein alpha subunit-palmitoylating enzyme. Mol Cell Biol 2009; 29: 435-447.

30. Pedram A, Razandi M, Deschenes RJ, Levin ER. DHHC-7 and -21 are palmitoylacyltransferases for sex steroid receptors. Mol Biol Cell 2012; 23: 188-199.

31. Rocks O, Gerauer M, Vartak N, Koch S, Huang ZP, Pechlivanis M et al. The palmitoylation machinery is a spatially organizing system for peripheral membrane proteins. Cell 2010; 141: 458-471.

32. Rossin A, Derouet M, Abdel-Sater F, Hueber AO. Palmitoylation of the TRAIL receptor DR4 confers an efficient TRAlL-induced cell death signalling. Biochem J 2009; 419: 185-192.

33. Oh Y, Jeon YJ, Hong GS, Kim I, Woo HN, Jung YK. Regulation in the targeting of TRAIL receptor 1 to cell surface via GODZ for TRAIL sensitivity in tumor cells. Cell Death Differ 2012; 19: 1196-1207.

34. Leon-Bollotte L, Subramaniam S, Cauvard O, Plenchette-Colas S, Paul C, Godard C et al. $\mathrm{S}$-nitrosylation of the death receptor fas promotes fas ligand-mediated apoptosis in cancer cells. Gastroenterology 2011; 140: 2009-2018.

35. Sharma C, Rabinovitz I, Hemler ME. Palmitoylation by DHHC3 is critical for the function, expression, and stability of integrin $\alpha 6 \beta 4$. Cell Mol Life Sci 2012; 69: 2233-2244.

36. Percherancier Y, Planchenault T, Valenzuela-Fernandez A, Virelizier JL, ArenzanaSeisdedos F, Bachelerie F. Palmitoylation-dependent control of degradation, life span, and membrane expression of the CCR5 receptor. J Biol Chem 2001; 276: 31936-31944.

37. Gao Z, Ni Y, Szabo G, Linden J. Palmitoylation of the recombinant human A1 adenosine receptor: enhanced proteolysis of palmitoylation-deficient mutant receptors. Biochem $\mathrm{J}$ 1999; 342: 387-395

38. Valdez-Taubas J, Pelham H. Swf1-dependent palmitoylation of the SNARE Tlg1 prevents its ubiquitination and degradation. EMBO J 2005; 24: 2524-2532.

39. Peli J, Schröter M, Rudaz C, Hahne M, Meyer C, Reichmann E et al. Oncogenic Ras inhibits Fas ligand-mediated apoptosis by downregulating the expression of Fas. EMBO J 1999; 18: 1824-1831.

40. Reichmann E. The biological role of the Fas/FasL system during tumor formation and progression. Semin Cancer Biol 2002; 12: 309-315.

41. Gaspar C, Cardoso J, Franken P, Molenaar L, Morreau H, Möslein G et al. Cross-species comparison of human and mouse intestinal polyps reveals conserved mechanisms in adenomatous polyposis coli (APC)-driven tumorigenesis. Am J Pathol 2008; 172: 1363-1380.

42. Drisdel RC, Green WN. Labeling and quantifying sites of protein palmitoylation. Biotechniques 2004; 36: 276-285.

Supplementary Information accompanies this paper on Cell Death and Differentiation website (http://www.nature.com/cdd) 